Gene expression profile of human lung epithelial cells chronically exposed to single-walled carbon nanotubes

Dongquan Chen

Todd A. Stueckle

Sudjit Luanpitpong

Yon Rojanasakul

Yongju Lu

See next page for additional authors 
Authors

Dongquan Chen, Todd A. Stueckle, Sudjit Luanpitpong, Yon Rojanasakul, Yongju Lu, and Liying Wang 


\title{
Gene expression profile of human lung epithelial cells chronically exposed to single-walled carbon nanotubes
}

\author{
Dongquan Chen ${ }^{1 *}$, Todd A Stueckle ${ }^{2}$, Sudjit Luanpitpong ${ }^{3}$, Yon Rojanasakul ${ }^{3}$, Yongju Lu ${ }^{4}$ and Liying Wang ${ }^{2^{*}}$
}

\begin{abstract}
A rapid increase in utility of engineered nanomaterials, including carbon nanotubes (CNTs), has raised a concern over their safety. Based on recent evidence from animal studies, pulmonary exposure of CNTs may lead to nanoparticle accumulation in the deep lung without effective clearance which could interact with local lung cells for a long period of time. Physicochemical similarities of CNTs to asbestos fibers may contribute to their asbestos-like carcinogenic potential after long-term exposure, which has not been well addressed. More studies are needed to identify and predict the carcinogenic potential and mechanisms for promoting their safe use. Our previous study reported a long-term in vitro exposure model for CNT carcinogenicity and showed that 6-month sub-chronic exposure of single-walled carbon nanotubes (SWCNT) causes malignant transformation of human lung epithelial cells. In addition, the transformed cells induced tumor formation in mice and exhibited an apoptosis resistant phenotype, a key characteristic of cancer cells. Although the potential role of p53 in the transformation process was identified, the underlying mechanisms of oncogenesis remain largely undefined. Here, we further examined the gene expression profile by using genome microarrays to profile molecular mechanisms of SWCNT oncogenesis. Based on differentially expressed genes, possible mechanisms of SWCNT-associated apoptosis resistance and oncogenesis were identified, which included activation of pAkt/p53/Bcl-2 signaling axis, increased gene expression of Ras family for cell cycle control, Dsh-mediated Notch 1, and downregulation of apoptotic genes BAX and Noxa. Activated immune responses were among the major changes of biological function. Our findings shed light on potential molecular mechanisms and signaling pathways involved in SWCNT oncogenic potential.
\end{abstract}

Keywords: Single-walled carbon nanotubes; Lung; Microarray; Gene expression; Pathways; p53

\section{Background}

Carbon nanotubes (CNT) are among the most widely used nanomaterials for various industrial and biomedical applications [1-3]. Their unique properties of high tensile strength, flexibility, thermal and electrical conductivity, and their light weight have contributed to their widespread use. However, their rapid growth in utility has raised a major concern about their safety, especially on human health and environment. CNT share several properties with asbestos fibers such as high aspect ratio, durability, biopersistence, and mode of exposure (e.g.,

\footnotetext{
* Correspondence: dongquan@uab.edu; Imw6@cdc.gov

'Division of Preventive Medicine, Department of Medicine, University of

Alabama at Birmingham, Birmingham, AL 35294, USA

${ }^{2}$ Pathology and Physiology Research Branch, National Institute for

Occupational Safety and Health, Morgantown, WV 26505, USA

Full list of author information is available at the end of the article
}

inhalation). Because asbestos fibers are classified as a Group I human carcinogen by the International Agency of Research in Cancer [4], there is a concern about the potential carcinogenicity of CNT [2]. Exposure to asbestos has been shown to cause various lung diseases including lung cancer and mesothelioma [5]. Thus, there is a clear need for carcinogenicity studies of CNT to develop control and prevention strategies [6].

Recent studies have shown that both single-walled (SW) and multi-walled (MW) CNT deposit in the deep lung tissue of mice with low clearance after pulmonary administration [7-9]. Occupationally relevant in vivo exposures (10 to $80 \mu \mathrm{g} /$ mouse) and pulmonary fibroblast in vitro exposure studies, using in vivo dose equivalents $\left(0.02\right.$ to $\left.0.2 \mu \mathrm{g} / \mathrm{cm}^{2}\right)$, resulted in dose-dependent transient pulmonary inflammation followed by fibroblast cell 
proliferation, alveolar wall thickening, and collagen I production culminating in persistent pulmonary fibrosis $[9,10]$. Several in vitro and in vivo studies have suggested the potential carcinogenicity of CNT [11-13]. The possible carcinogenic mechanisms include DNA damage [14-16], mitotic disruption [12], impaired apoptosis, and activation of oncogenic signaling events, which recapitulate asbestos-induced lung cancer and mesothelioma [17-19]. It was suggested that nanotube bundles are similar in size to microtubules that form the mitotic spindle and may be incorporated into the mitotic spindle apparatus, resulting in multipolar mitosis and aneuploidy [20] which were observed in asbestos-treated cells [15]. Furthermore, CNT exposure induced asbestos-like granulomas in mice in a short-term abdominal instillation study [18].

Multiple genes may be involved in single-walled carbon nanotubes (SWCNT) pathogenesis. For examples, p38 MAPK was reported to regulate SWCNT-induced fibrogenic and angiogenic responses [21]. Other shortterm exposure studies report increased NF-k $\beta$, TNF $\alpha$, NRLP3, iNOS, p53, and TGF $\beta$ activity all culminating in lung inflammation and the onset of pulmonary interstitial fibrosis [22,23]. Long-term in vitro and in vivo exposure studies found that SWCNT and MWCNT exposure to lung tissues resulted in KRAS activation, enhanced micronuclei and nuclear protrusions, mitosis disruption, mutated p16, cMyc proto-oncogene signaling, and enhanced metalloproteinase remodeling of the extracellular matrix [9,24-27], which all indicate tumorigenesis potential following CNT exposure. Recent transcriptomic and sequencing studies suggest that long-term CNT exposure results in similar signaling compared to other biopersistent fibers (i.e., asbestos) but also display unique signaling pathways [27-30] raising the possibility of a distinct $\mathrm{CNT}$ pathogenesis paradigm [31]. Although a majority of these proinflammation and pro-fibrotic genes have known roles in cancer, a consensus on those key molecules and signaling pathways that potentially drive and contribute to CNT-associated carcinogenesis remain elusive.

Our recent studies showed that low-dose $\left(0.02 \mu \mathrm{g} / \mathrm{cm}^{2}\right)$ SWCNT exposure caused malignant transformation and tumorigenesis of human lung bronchial epithelial cells [13]. We also found that phosphorylation at various positions on p53 in these malignant cells significantly decreased after sub-chronic SWCNT exposure [13]. These phosphorylation sites are crucial for stabilization and activation of p53-dependent functions of tumor suppression, DNA damage repair, and apoptosis resistance [32-35]. At present, the upstream and downstream signal transduction pathways associated with altered p53 status contributing to a SWCNT-induced epithelial tumorigenic phenotype are unknown.
Since carcinogenesis is a long-term, multi-step process and involves multiple genes, it is of critical importance to study the chronic exposure and genome-wide expression changes to define the underlying molecular mechanisms of carcinogenesis, which is largely unknown. To accomplish this goal, we have developed a sub-chronic exposure model in which human lung bronchial epithelial cells, the primary target of CNT inhalation exposure, were continuously exposed to low-dose SWCNT in culture over a long period (6 months) [13]. Because isolated primary lung cells do not survive in long-term culture, we employed an immortalized human lung epithelial BEAS-2B cell line which is non-tumorigenic and exhibits similar cellular responses $[7,20,36]$. The longterm SWCNT-exposed BEAS-2B (B-SWCNT) cells were evaluated, for the first time, for genome-wide expression profiling and functional analysis compared to unexposed, passage-matched control cells. Our study revealed multiple genes and signaling networks that are affected by SWCNT-induced oncogenic transformation which might serve as potential SWCNT-specific exposure or disease markers. This in vitro approach also supports prudent adoption of exposure control strategies protection of workers, consumers, and the environment.

\section{Methods}

\section{Preparation of single-walled carbon nanotubes}

SWCNT (CNI, Houston, TX, USA) were produced by the high-pressure $\mathrm{CO}$ disproportionation (HiPco) technique, employing $\mathrm{CO}$ in a continuous-flow gas phase as the carbon feedstock and $\mathrm{Fe}(\mathrm{CO})_{5}$ as the iron-containing catalyst precursor and used in our previous study [13]. Briefly, the SWCNT were purified by acid treatment to remove metal contaminates. Elemental analysis of the supplied SWCNT showed that the SWCNT were 99\% elemental carbon and $0.23 \%$ iron. The specific surface area measured at $-196^{\circ} \mathrm{C}$ by the nitrogen absorptiondesorption technique (Brunauer-Emmet-Teller method) was 400 to $1,000 \mathrm{~m}^{2} / \mathrm{g}$. The diameter and length distribution of the SWCNT measured by field emission scanning electron microscopy which were 0.8 to $1.2 \mathrm{~nm}$ and 0.1 to $1 \mu \mathrm{m}$, respectively. SWCNT were dispersed by acetone/sonication method as previously described [10]. Briefly, SWCNT were treated with acetone and placed in an ultrasonic bath for $24 \mathrm{~h}$. The dispersed CNT were then filtered from the solution using a $20-\mu \mathrm{m}$ nylon mesh screen followed by a $0.2-\mu \mathrm{m}$ polytetrafluoroethylene filter. After filter collection, the dispersed CNT were washed thoroughly with distilled water and suspended in phosphate-buffered saline (PBS) with 2- to 3-min sonication (Sonic Vibra Cell Sonicator, Sonic \& Material Inc, Newtown, CT, USA). 


\section{In vitro treatment and cell sample preparation for microarrays}

Microarray sample collection and analyses were performed on unexposed and SWCNT-exposed cells from our previous study [13]. Briefly, human lung bronchial epithelial BEAS-2B cells were continuously exposed for 6 months to a sub-cytotoxic concentration $\left(0.02 \mu \mathrm{g} / \mathrm{cm}^{2}\right.$; equivalent to $0.1 \mu \mathrm{g} / \mathrm{ml}$ ) of dispersed SWCNT or PBS (non-treatment control) in a six-well culture plate in triplicate. Each replicate was exposed and independently assayed throughout the study. This relatively low concentration was chosen due to its relevance to in vivo SWCNT exposure dose of $10 \mu \mathrm{g} /$ mouse previously reported $[8,37,38]$. Repeated, long-term dosing of particulate in in vitro systems may result in accumulation of particle burden over time in the assay system. To reduce and safeguard against potential SWCNT accumulation over time, every 3 to 4 days, the exposure media was aspirated and the cells were triplicate washed with PBS to remove suspended and unbound SWCNT. Cells were then resupplied with new exposure media containing SWCNT. The cells were passaged weekly at preconfluent densities using a solution containing $0.05 \%$ $w / v$ trypsin and $0.5 \mathrm{mM}$ EDTA (Invitrogen, Carlsbad, CA, USA). Greater than $50 \%$ of cells were removed during passaging which resulted in a relatively small proportion of intracellular SWCNT remaining in the system. Parallel cell cultures grown in the same media without SWCNT provided passage-matched control. Following the 6-month exposure, SWCNT-treated (B-SWCNT) and control (B-Control) cells were evaluated for genome-wide gene expression. Three B-SWCNT cell biological replicates showing similar phenotypic behavior and p53 expression patterns [13] were pooled and analyzed as one. Additional filters such as increasing the fold change to $>2$ will limit this disadvantage. Since the objective of this study was to explore the candidate genes involved in the carcinogenic process for further studies, this study is considered a preliminary step.

RNA sample preparation and hybridization were performed as previously described [39]. Briefly, total RNA was harvested and isolated using TRIzol (Invitrogen) according to manufacturer's instructions. Isolated RNA in DEPC-treated water was then immediately frozen at $-80^{\circ} \mathrm{C}$ for $24 \mathrm{~h}$ and shipped on dry ice to ArrayStar (Rockville, MD, USA) for mRNA processing, microarray hybridization, and probe expression normalization.

\section{DNA microarray and data analysis}

Whole genome oligo microarrays (\#014850, Agilent Technology, Santa Clara, CA, USA) were used. Each array represents more than 41,000 unique human genes and transcripts sourced from RefSeq, Goldenpath Ensembl Unigene Human Genome (Build 33) and GenBank databases.
Fluorescent RNA targets were prepared using Agilent Quick Amp Labeling Kit. Microarray hybridization was performed at $65^{\circ} \mathrm{C}$ for $17 \mathrm{~h}$ in Agilent's SureHyb hybridization chambers. After washing in an ozonefree environment, the slides were scanned using the Agilent DNA microarray scanner (model G2505B).

Raw data were extracted using Agilent Feature Extraction Software (Santa Clara, CA, USA). Expression raw data from passage control cells were used for other exposure studies. The resulting text files were imported into the Partek Genomic Suite (PGS; St. Louis, MO, USA) for preprocessing, normalization, and statistical analysis. Briefly, normalization was performed using the Agilent FE one-color scenario (mainly median normalization). Genes marked present or marginal in all samples ('All Targets Value') were chosen for further data analysis. Differentially expressed genes (DEGs) were identified through fold-change screening and $t$-test assuming unequal variances. Genes were differentially expressed in B-SWCNT cells if they exhibited $\geq \pm$ twofold expression and $p \leq 0.05$ compared to B-Control cells. Unsupervised hierarchical clustering, gene ontology (GO) analysis, and pathway analysis were performed using the PGS (St. Louis, MO). False discovery rate (FDR) assessment by Benjimini Hochberg methods was applied for multiple hypothesis testing purpose [40]. The filtered gene lists were used for GO, pathways, and gene-gene interaction analyses by using Ingenuity Pathway Analysis (IPA) software package (Redwood City, CA, USA).

\section{Apoptosis protein array}

To substantiate the genomic data and further identify proteins contributing to the apoptotic resistance phenotype, B-SWCNT and B-Control cell protein lysates were subjected to a human apoptosis protein expression array (see Additional file 1: Table S1 for array layout) in duplicate following manufacturer's instructions ( $R$ \& D Systems, Minneapolis, MN, USA). Briefly, $2 \times 10^{6}$ cells from each treatment were seeded into a $25-\mathrm{cm}^{2}$ flask in particle-free growth medium and cultured for $24 \mathrm{~h}$. Next, plated cells were placed on ice, washed in ice cold PBS, and lysed using the manufacturer's lysis buffer. Cell lysate was collected via rubber policemen, rocked on ice in suspension of $30 \mathrm{~min}$, and spun at $12.5 \mathrm{~K} \times g$ to collect supernatant. Protein levels were determined via BCA absorbance method on a spectrophotometer at $562 \mathrm{~nm}$. Five hundred micrograms of protein was incubated with each protein array overnight at $4^{\circ} \mathrm{C}$. Each array was triplicate washed in buffer, incubated with detection antibody for $1 \mathrm{~h}$, washed again, and incubated with streptavidin-HRP antibody for $30 \mathrm{~min}$. Protein expression was captured using HRP chemiluminescence on X-ray film. 


\section{Western blot}

$\mathrm{Bcl}-2$, pAkt, and Akt protein expression was determined using previously described Western blot methods [10]. Briefly, B-SWCNT and B-Control cells were seeded $\left(5 \times 10^{5}\right.$ cells/well $)$ and cultured in six-well plates for $24 \mathrm{~h}$ in particle-free medium or re-exposed to dispersed SWCNT ( 1 to $50 \mu \mathrm{g} / \mathrm{ml}$ or 0.1 to $5.2 \mu \mathrm{g} / \mathrm{cm}^{2}$ ). Cells were placed on ice, washed with ice cold PBS buffer, and lysed in protein buffer containing PMSF and protease inhibitor (Invitrogen). Cells were scraped, collected, and rocked on ice for $30 \mathrm{~min}$. Following centrifugation at $12.5 \mathrm{~K} \times g$, BCA assay was used to measure protein concentration in collected supernatants. Equal amounts of protein were loaded into SDS PAGE gel, allowed to separate under current, and transferred to PVDF membranes using semi-dry transfer apparatus (Fisher Scientific, Pittsburgh, PA, USA). Membranes were incubated with antibodies to either Bcl-2, pAkt, Akt (Cell Signaling, Beverly, MA, USA), or B-actin (Sigma, St. Louis, MO, USA) overnight at $4^{\circ} \mathrm{C}$, washed thrice in Tris buffer with $0.1 \%$ Tween, and incubated with anti-rabbit or anti-mouse HRP antibody for $1 \mathrm{~h}$ (Santa Cruz, Inc, Paso Robles, CA, USA). Protein levels were determined using Millipore Immobilon HRP chemiluminescent substrate (Millipore, Billerica, MA, USA) and exposure to Xray film. Western blot experiments were independently performed three times. Quantification of Western blot results were performed by using ImageJ [41,42]. Statistical analyses were performed by using two group Student $t$-tests after quantification and normalization to corresponding controls.

\section{Results and discussion}

As we reported previously [13], transformed B-SWCNT cells displayed aberrant p53 signaling, increased aggressiveness, apoptosis resistance, anchorage-independent growth, angiogenicity, and in vivo xenograft tumor formation compared to unexposed passage control cells. These findings suggested the potential carcinogenicity of B-SWCNT cells. The present transcriptomic analysis and supporting data also indicate aberrant p53 signaling, pAkt, Ras, Notch 1, and altered intrinsic mitochondrial pathway signaling as potential mechanisms for SWCNT carcinogenicity. This is consistent with the previous observations that most human cancers possess p53 tumor suppressor gene inactivation resulting in dysfunctional signaling contributing to tumorigenesis $[43,44]$.

\section{Gene expression profile by hierarchical clustering}

Hierarchical clustering is a convenient way to visualize the overall similarity among a large pool of samples. The heatmap pattern also offers clues of possible coexpression of genes. As shown in Figure 1, three control samples showed similar gene expression patterns

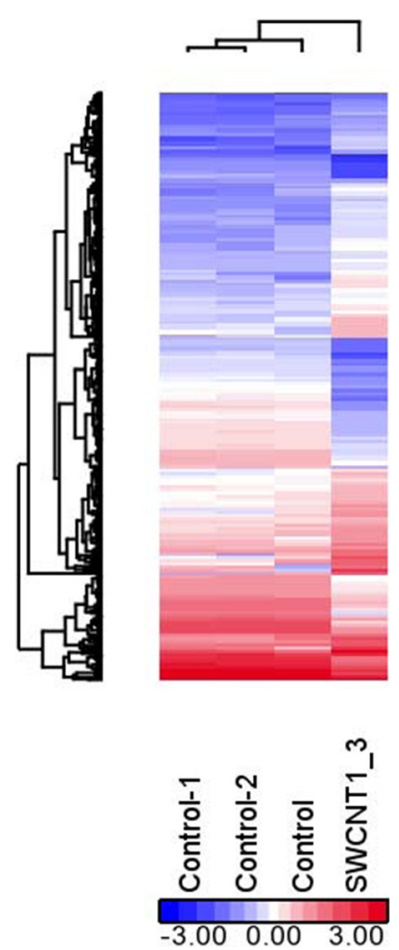

Figure 1 Hierarchical clustering of SWCNT-exposed and passage control cell gene expression following 6 months of continuous treatment. The hierarchical clustering was based on differentially expressed genes of SWCNT vs. control comparison using $p<0.05$ and fold changes $>2$ criteria. Color indicates log2-transformed normalized intensities with red and blue indicating over- and underexpression, respectively.

to cluster on the left (control, control-1, and control-2). The B-SWCNT cells possessed a different expression pattern on the right (SWCNT1_3). Here, three treated samples were pooled and analyzed as one. This is a costeffective way to get the most likely candidate genes but with the disadvantage of lowering the power and introducing more false positives. Since the objective of this study was to explore the candidate genes involved in the carcinogenic process for further studies, this study is considered a preliminary step.

\section{Gene ontology (GO) analysis for biological processes and} molecular functions

We analyzed top GO biological processes that are affected by SWCNT exposure based on the list of DEGs in B-SWCNT cells. Genes that are involved in immune response were among the top biological processes (Table 1). The top biological processes include the responses to metal ion, antigen process and presenting, regulation of phagocytosis, and vacuolar transport. Apparently, lung epithelial cells may recognize SWCNT as foreign which can alter the innate immune response and influence the immune system as reported $[27,45,46]$. 
Table 1 Top biological processes changed after SWCNT treatment in transformed BEAS-2B cells compared to unexposed cells

\begin{tabular}{|c|c|c|c|c|}
\hline Biological process & Enrichment score & Enrichment $p$ value & $\begin{array}{l}\text { Percent of genes in group } \\
\text { that are present }\end{array}$ & GO ID \\
\hline Response to metal ion & 8.6 & 0.000 & 37.5 & 10038 \\
\hline $\begin{array}{l}\text { Antigen processing and presentation of peptide } \\
\text { antigen via MHC class I }\end{array}$ & 8.5 & 0.000 & 8.6 & 2474 \\
\hline Regulation of circadian rhythm & 7.9 & 0.000 & 30 & 42752 \\
\hline Antigen processing and presentation & 7.4 & 0.001 & 10.9 & 19882 \\
\hline Positive regulation of microtubule depolymerization & 7.3 & 0.001 & 66.7 & 31117 \\
\hline Regulation of phagocytosis & 7.3 & 0.001 & 66.7 & 50764 \\
\hline Interspecies interaction between organisms & 7 & 0.001 & 4.2 & 44419 \\
\hline Cellular chloride ion homeostasis & 6.6 & 0.001 & 50 & 30644 \\
\hline Vacuolar transport & 6.6 & 0.001 & 50 & 7034 \\
\hline Rhythmic process & 6.4 & 0.002 & 18.8 & 48511 \\
\hline
\end{tabular}

The biological process was based on SWCNT vs. control comparison, $p<0.05$ and fold changes $>2$.

In addition, the positive regulation of microtubule depolymerization suggests that long-term SWCNT exposure altered microtubule dynamics as previously reported [12,47]. Similarly, genome wide changes of GO molecular functions after SWCNT exposure included genes responsible for immune response (i.e., MHC class I) protein binding, post-transcriptional modifications (e.g., ubiquitin protease activity), and other enzymatic activities were among the top molecular function changes (Table 2). Previous 'omics' studies using long-term SWCNT exposure in vivo or in vitro models reported similar findings that protein posttranslational modification constitutes a large GO signal $[27,28]$ and is likely to play a large role in disease development and pathology.

\section{Functional analysis of gene-gene interactions}

Multiple gene-gene interactions are important for gene functions and signal transduction. Therefore, gene-gene interaction networks were plotted, scored, and ranked to identify interactions potentially driving the observed BSWCNT malignant phenotype. Consistent with the observed carcinogenic and apoptosis-resistant phenotype of B-SWCNT cells [13], immune response, cell growth, cell death/survival, and cell cycle control signaling network were among the major functions identified in the top-ranked networks in the B-SWCNT toxicogenomic profile (Table 3). The functional involvement of individual genes or pathways in the carcinogenic process needs to be further studied individually to determine their actual involvement. Regardless, the pathways of Cellular growth and proliferation, hematological system development and function, humoral immune response, cell death and survival, embryonic development, organismal development, and cancer-related gene-gene interaction networks possessed gene-gene interactions known to participate in tumorigenic and CNT-induced neoplastic signaling mechanisms [27].

Table 2 Top molecular functions changed after SWCNT treatment in transformed BEAS-2B cells

\begin{tabular}{lllll}
\hline Molecular function & Enrichment score & Enrichment $\boldsymbol{p}$ value & $\begin{array}{l}\text { Percent of genes in group } \\
\text { that are present }\end{array}$ \\
\hline MHC class I receptor activity & 13.6 & 0.000 & 35.7 & 32393 \\
Flavonol 3-sulfotransferase activity & 7.3 & 0.001 & 66.7 & 47894 \\
Aryl sulfotransferase activity & 6.1 & 0.002 & 40.0 & 4062 \\
G-protein coupled photoreceptor activity & 6.1 & 0.002 & 40.0 & 5020 \\
SH3 domain binding & 6.1 & 0.002 & 5.8 & 17124 \\
Ubiquitin-specific protease activity & 5.7 & 0.003 & 22.2 & 4843 \\
Photoreceptor activity & 4.9 & 0.008 & 1.9 & 9881 \\
Protein binding & 4.7 & 0.009 & 5.8 & 4515 \\
Ubiquitin thiolesterase activity & 4.7 & 0.009 & 6.9 & 4197 \\
Cysteine-type endopeptidase activity & 4.5 & 0.011 & & 4221 \\
\hline
\end{tabular}

The molecular function was based on SWCNT vs. control comparison, $p<0.05$ and fold changes $>2$. 
Table 3 Top affected gene-gene interaction networks after sub-chronic SWCNT exposure in transformed BEAS-2B cells

Top diseases and functions
Cellular growth and proliferation, hematological
system development and function, humoral immune
response

Connective tissue disorders, inflammatory disease, skeletal and muscular disorders

Molecules in network ${ }^{\mathbf{a}, \mathbf{b}}$
ADAM15, ADRBK2, ATP5B, BCL10, BCR (complex), CHGA, CR2, CRHR1,
ERK1/2, FGR, GNAZ, HOPX, HSD11B2, HSPD1, HTATIP2, IgG1, Igg3, IgG2b,
IGLL1/IGLL5, Igm, insulin, ITCH, Jnk, MFGE8, NEU1, PAK2, PAX4, PPP1R1
Ras, RASGRP1, SH2B2, SH2D2A, TAS1R1, Vegf, ZNF24
C1R, CD33, CD59, CDON, G6PD, HLA-A, HLA-B, HLA-C, HLA-E, Ifnar, IgG,
IKK (complex), immunoglobulin, interferon alpha, KIR, KRT31, MYO1E,
NFAT5, NFkB (complex), P38 MAPK, PI3K (family), PIM1, PkC(S), RHOB,
RNF34, RTKN, SCARB1, SIRPA, SLC6A6, Tap, TAPBP, TNFSF10, TRAF3IP2,
USP11, USP18

ADAM17, AFF4, AKT2, Akt, AMPK, Ap1, BHLHE40, CD68, CELF1, CSNK1E, CSNK1G2, Cyclin A, DVL1, estrogen receptor, F actin, FMR1, GSK3B, HMGB3, HMGN5, Hsp70, IGFBP2, LCP1, LDL, MGLL, MUC4, OSGIN1, PDGF BB, PI3K (complex), PMAIP1, Ppp2C, PURA, SF3B3, STRN, USP6, USP33

Cellular compromise, skeletal and muscular system development and function, post-translational modification

Cell morphology, cellular assembly and organization, cellular development

Respiratory system development and function, tissue morphology, cell cycle

Cancer, gastrointestinal disease, hepatic system disease

Cell morphology, cell-to-cell signaling and interaction nervous system development and function

DNA replication, recombination, and repair, cellular compromise, cell death and survival

ARHGEF9, ATP13A2, CD3, Cg, Creb, CTSB, DUSP9, ERK, FBN1, FGF3, FSHR, GSTM4, Hdac, Histone h3, Histone h4, HSD17B1, IFI44, IFI44L, IgG2a, KDM3A, Lh, MCOLN1, MT1F, MT1X, NDRG1, NDRG4, NES, NPNT, ORAI1, OXT, PGK1, SOX12, STAT5a/b, STK17A, TCR

ACBD7, ADAM22, ANK1, AR, AS3MT, ATP5S, ATP6V0E2, ATP8B1, CACNA1E, CDC6, CHD8, DLG4, FARP1, GLUL, GPR182, GSTM1, HILPDA, HIST1H1A, IGF1R, KCNA4, KCNAB2, MCM8, NIPSNAP1, NUPR1, PPT2, RAB18, RCN2, SIPA1L1, SOX11, ST6GALNAC6, SYNGAP1, TEX2, TMEM30A, WNK1, ZSCAN16

26 s Proteasome, ACTC1, ACTN1, ACTR2, ACTR3, BNIP3L, CAP2, CARD10, CKAP2, EBAG9, ELMOD3, ENC1, FAM89B, FSH, GEM, IGLL1/IGLL5, ING2, JMJD6, MED26, MT1L, NOL3, NR3C1, PRPH, PSMB1, RAB11A, RDM1, RNA polymerase II, SCAF8, SERTAD2, SFTPC, SIAH2, SMARCC1, TFDP2, TUBA4A, USP13

ACOT11, AHCY, ANXA7, ARL6IP1, ARNT, ASS1, AXIN1, AXIN2, BCLAF1, CAMLG, CCDC80, CYP1A1, EPHX1, FAM120A, FBXW7, HIST1H1C, IGFBP2, KAT5, KITLG, KREMEN2, MED13L, MT1H, N-cor, NCOR2, NQO1, PIAS2, PSMF1, PTGDS, PTP4A3, RBBP4, SERPINB6, SIDT2, STOX1, TCF7L2, TP53

AIG1, ALDH3B1, C11 orf30, CABYR, CACNA2D1, CHMP4B, COX10, CYFIP2, DGKZ, DMD, DNAJB6, DTNB, DYRK1A, HDAC4, HYOU1, INS, LEPR, Map4k4, MARK3, OPN4, Pdx1, PFKM, PPFIA1, proinsulin, SNCA, SNTB2, SPARC, SPOP, TNRC6B, TRIM44, TUBB4A, VEGFA, VGF, YWHAG, YWHAH

ATP6VOE1, BAG1, BAX, BGN, BRCA1, BRCA2, CCND1, CEL, CKB, DDX5, DNAJA1, DPYSL3, FANCD2, GATA1, GFI1B, GSK3A, H3F3A/H3F3B, Hbb-b2, HSPA4, HSPD1, LATS1, LINC00467, MAPT, NGFR, NME4, OPA1, ROCK2, SFN, SPOCK2, STIP1, TLE1, UBE2N, USP11, ZFPM1, ZNF324

Lipid metabolism, molecular transport, small molecule biochemistry

AP1M2, APOA1, APOC1, ARCN1, CBS, CCL1, CCL22, CETP, CLIC4, COG3, COPG1, COPZ1, CXCL5, ERN1, EYA4, GSTA1, HLA-J, HYOU1, IFNB1, LCAT, MGLL, NPEPPS, Pdx1, PEX6, PHLDB2, PPARG, PTGES2, RGS14, RNASE1, TBXAS1, TMEM173, TNF, TPM2, Trim30a/Trim30d, XBP1

Score Focus

molecules ${ }^{c}$

35

25

${ }^{a}$ The network was based on SWCNT vs. control comparison, $p<0.05$ and fold changes $>2$.

${ }^{b}$ Capitalized names indicate genes while lower case names indicate complexes.

'The number of differentially expressed genes in each network are reported.

\section{Aberrant p53 signaling}

Our initial study reported B-SWCNT malignant transformed cells possessing an apoptotic resistant phenotype due in part to aberrant p53 signaling [13]. Apoptosis plays an essential role in the removal of mutated or transformed cells and its disruption contributes to abnormal cell growth and malignancy $[48,49]$. To expand on this finding, we first examined p53 (TP53) and p53associated protein mRNA expression in B-SWCNT compared to B-Control cells. Moderate decreased expression of TP53 mRNA was observed, but no statistical significance was found (Table 4). Although 13 other p53-associated transcripts showed no statistical difference, three transcripts experienced increased or decreased expression (>twofold) which included TP53I3, TP53INP1, and TP53INP2. This data suggested a moderate alteration of TP53-related signaling potentially due to post-transcriptional modifications. Overexpressed TP5313, an oxidoreductase, is typically observed as a response to reactive oxidative species (ROS) stress which has been shown to occur in B-SWCNT cells $[10,50]$. More interestingly, decreased TP53INP1 and TP53INP2 
Table 4 mRNA expressions of TP53 and related genes in SWCNT-transformed BEAS-2B cells

\begin{tabular}{|c|c|c|c|c|}
\hline Probe ID & Gene & Description & $p$ value & Fold \\
\hline A_23_P26810 & TP53 & Homo sapiens tumor protein p53 (Li-Fraumeni syndrome) & 0.66 & -1.48 \\
\hline A_24_P274842 & TP53AP1 & Homo sapiens mRNA for P53TG1-B, complete cds. & 0.75 & 1.26 \\
\hline A_23_P145895 & TP53AP1 & Homo sapiens mRNA for P53TG1-C, complete cds. & 0.94 & 1.07 \\
\hline A_23_P88703 & TP53BP1 & Homo sapiens tumor protein p53 binding protein, 1 & 0.70 & -1.21 \\
\hline A_23_P12526 & TP53BP2 & Homo sapiens tumor protein p53 binding protein, 2, transcript variant 2 & 0.17 & -1.50 \\
\hline A_23_P150281 & TP53111 & Homo sapiens tumor protein p53 inducible protein 11 & 0.82 & -1.75 \\
\hline A_24_P185207 & TP53113 & Homo sapiens tumor protein p53 inducible protein 13 & 0.55 & 1.61 \\
\hline A_24_P185205 & TP53113 & Homo sapiens tumor protein p53 inducible protein 13 & 0.68 & 1.44 \\
\hline A_23_P5392 & TP5313 & Homo sapiens tumor protein p53 inducible protein 3, transcript variant 1 & 0.18 & 2.55 \\
\hline A_23_P168882 & TP53INP1 & Homo sapiens tumor protein p53 inducible nuclear protein 1 & 0.10 & -4.86 \\
\hline A_24_P357465 & TP53INP2 & Homo sapiens tumor protein p53 inducible nuclear protein 2 & 0.53 & -2.66 \\
\hline A_24_P245646 & TP53RK & Homo sapiens TP53 regulating kinase & 0.60 & -1.33 \\
\hline A_24_P227971 & TP53TG3 & Homo sapiens TP53TG3 protein & 0.65 & 1.50 \\
\hline A_23_P49391 & TP53TG3 & Homo sapiens TP53TG3 protein & 0.95 & 1.07 \\
\hline
\end{tabular}

expression suggests a decreased ability in B-SWCNT cells to respond to cell stress. Both genes encode for antioxidant proteins that respond to cell stress by positively regulating autophagy and directly phosphorylating serine 46 on p53 protein which is known to increase p53's apoptotic signaling ability [33]. BEAS-2B cells exhibit increased autophagy in response to ROS stress, including CNT and other inhalable xenobiotic exposure, and dysregulation of autophagy may contribute to tumor promotion potential [50,51]. Decreased expression of these two transcripts suggests that B-SWCNT cells lost two major cell protection mechanisms including a major p53 pro-apoptotic signaling pathway and positive regulation of autophagy.

To identify pathways that potentially altered p53 signaling and fate, several canonical pathways with DEGs with known p53 functions were plotted in IPA. Upregulation of PTEN and lowered expression of AKT2 suggested decreased Akt signaling upstream of MDM2 regulation of p53 (Figure 2). Also, decreased GSK3 $\beta$ expression suggests release of $\beta$-catenin from APC complex resulting in activation of Wnt signaling [52] and a potential block to MDM2-mediated p53 degradation via the proteosome.

To substantiate post-translational phosphorylation of p53 and identify other proteins contributing to BSWCNT cell apoptotic resistance ability, we conducted an apoptosis protein expression array. Figure 3 shows, in addition to substantial decreased phosphorylation of Ser46, a target of TP53INP1/2, Ser15 and Ser392, targets of MDM2 and casein kinase II [13,33,53], which indicated an unstable and dysfunctional p53 response in contributing to B-SWCNT malignant phenotype and enhanced cancer signaling (Table 3, network 7). Other apoptotic- associated proteins were found to alter expression. A substantial decrease in claspin expression indicated loss of checkpoint-mediated ATR control of the cell cycle following DNA damage. Overexpressed heat shock protein 60 (HSPD1) in B-SWCNT cells may contribute to cell proliferation and apoptotic resistance in response to genotoxic stress (Table 3, networks 1 and 9) by stabilizing, surviving, and inhibiting p53 [54]. Moderate decreased TNFR1, the major receptor for TNF $\alpha$, indicated a reduced ability to respond to pro-death TNF $\alpha$ signal which was previously confirmed [13]. Conversely, a fivefold overexpressed Fas receptor in the extrinsic apoptotic pathway suggested a sensitization to apoptosis; however, it is possible that downstream modulators of Caspase 8 activity (i.e., cFLIP) may block this effect. Bcl-xl and Xiap, anti-apoptotic Bcl-2 family proteins, experienced a moderate decrease (twofold) in B-SWCNT compared to B-Control cells. Cells undergoing tumorigenic transformation typically exhibit changes in both pro-survival and death proteins [49]. Investigation of both extrinsic and intrinsic signaling pathways and their role in B-SWCNT apoptotic resistance is currently under investigation in our group.

A recent demonstration of MWCNT-induced mesothelioma in p53 knockout mice [19,55] further supports our finding and the role of p53 in CNT carcinogenesis. A portion of this response may be due to a weakened p53 in the BEAS-2B cell model since the SV40 large T antigen immortalization procedure does affect $\mathrm{p} 53$ function [44]. Numerous studies acknowledge that p53 status is a contributing factor to adverse effects following many different types of inhalable and respirable particle exposure to the lung $[34,36,38,39,43]$. In addition, other studies note that a CNT's physicochemical properties and toxicokinetics in combination with p53 status of exposed 
Extracellular space

Cytoplasm

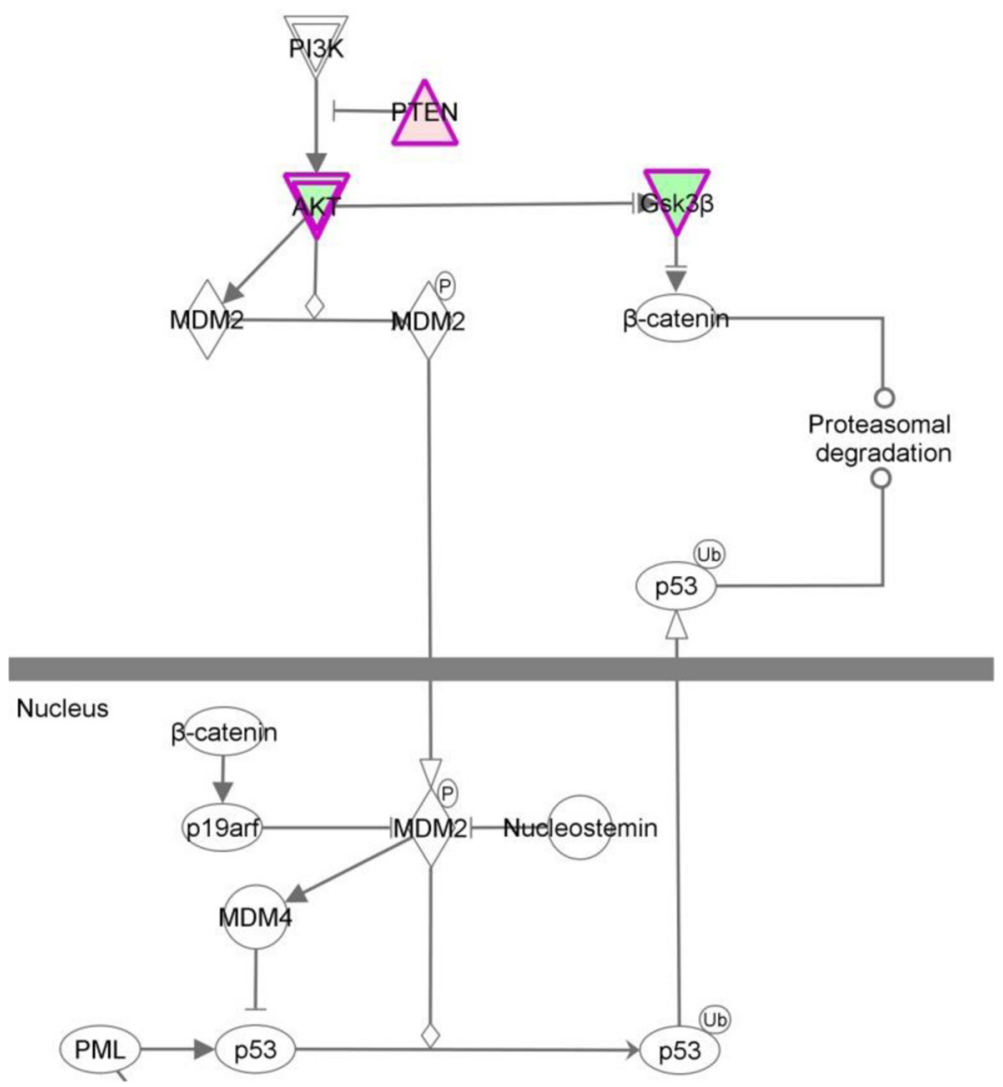

Figure 2 Akt2, PTEN, and GSK3B-mediated alteration of p53 and $\beta$-catenin function in SWCNT transformed BEAS-2B cells. Color indicates upregulation (in red) and downregulation (in green). Protein assay (Figure 4) showed no significant level changes of Akt proteins and increased phosphorylated Akt indicating that activated Akt may play a role in Gsk3ß inhibition of B-catenin and MDM2-mediated p53.

A

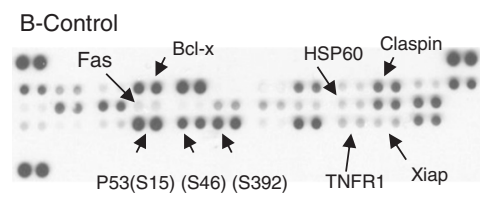

B-SWCNT

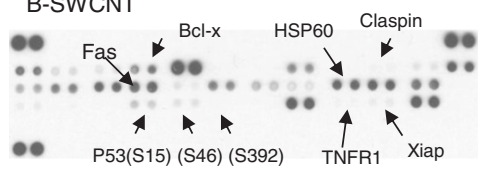

B

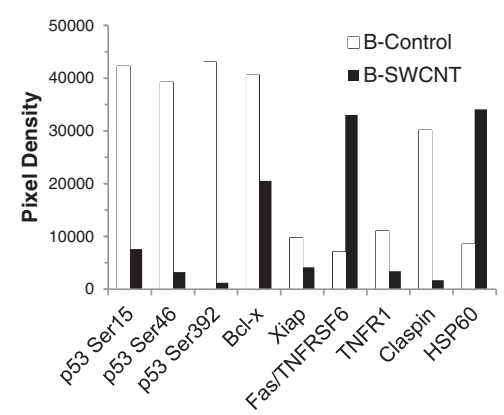

Figure 3 Apoptosis protein expression array comparison of B-SWCNT to B-Control cells. Apoptosis protein expression array comparison of B-SWCNT to B-Control cells showed (A) altered BCl-x, Xiap, Fas, TNFR1, claspin, and HSP60 expression in addition to three serine phosphorylation sites on p53 shown in our previous report [13]. (B) Densitometry quantification of protein array. Representative data are shown from two independent replicate experiments. See Additional file 1: Table S1 for array layout. 
tissue determine disease response [56,57]. Regardless, SWCNT exposure did alter p53 signaling in these cells compared to B-Control cells. These findings add to the growing literature that pre-existing p53 condition along with physicochemical properties of CNTs contribute to disease outcome following respirable fiber exposure [19].

Since the above evidence suggested MDM2 activity and $A K T 1$ is a major signaling protein in most cells compared to $A K T 2$, we investigated whether $\mathrm{B}-\mathrm{SWCNT}$ cells compared to B-Control cells exhibited increased AKT activation via phosphorylation, both in the presence and absence of SWCNT. Western blot analysis indicated increased ratio (1.7-fold, $p<0.05)$ of phosphorylated Akt (pAkt) to Akt protein expression in 0 to $5 \mu \mathrm{g} / \mathrm{ml} \mathrm{SWCNT}$ in B-SWCNT compared to B-Control cells (Figure 4A,B). Exposure to SWCNT caused a moderate $20 \%$ increase in pAkt levels in B-Control cells at $\geq 10 \mu \mathrm{g} / \mathrm{ml}$ after $12 \mathrm{~h}$ exposure while re-exposure to B-SWCNT exhibited a $27 \%$ decrease at high dose levels $(\geq 25 \mu \mathrm{g} / \mathrm{ml})$. These results show that unexposed lung epithelial cells experience enhanced Akt activation following SWCNT exposure [17]. In addition, it suggested that prolonged, low-dose SWCNT exposure results in a dysregulated, constitutively active PAKT and that Akt signaling may not be regulated at the mRNA level. Akt activation, in turn, potentially activated MDM2, leading to enhanced p53 ubiquitination and potential blockage of GSK3 $\beta$ (Figure 2). Thus, PI3K-AKT signaling may be involved in SWCNT oncogenesis by promoting cell survival (Table 3, network 3). Since pAkt is known to suppress intrinsic mitochondrial pathway and p53 blocks Bcl-2 expression, protein analysis showed that Bcl-2 protein expression was significantly elevated 2.5 -fold in B-SWCNT compared to B-Control cells at $0 \mu \mathrm{g} / \mathrm{ml}$ $(p<0.05$; Figure $4 \mathrm{~A}, \mathrm{C})$. At $12 \mathrm{~h}$ post-exposure to SWCNT, neither cell type displayed a significant change in Bcl-2 expression. Following 24-h SWCNT exposure, B-Control cells displayed a significant increase in $\mathrm{Bcl}-2$ expression at $\geq 5 \mu \mathrm{g} / \mathrm{ml}$. Conversely, B-SWCNT displayed a significant decrease in Bcl-2 expression at $\geq 10 \mu \mathrm{g} / \mathrm{ml}$. These dosedependent $\mathrm{Bcl}-2$ expression changes correlated with pAkt levels in each cell type. This suggested that SWCNT exposure to lung epithelial cells first results in Akt activation at $12 \mathrm{~h}$ followed by increased Bcl-2 at $24 \mathrm{~h}$, which potentially occurs via either diminished p53 signaling to Noxa [33] or blockage of BAD activity [48]. Persistent stimulation of this pathway could result in an apoptosis resistance phenotype via $\mathrm{Bcl}-2$ overexpression. Interestingly, the dose-dependent drop in pAkt expression mirrored the drop in Bcl-2 expression (-28\%) in re-exposed B-SWCNT cells, which resulted in equivalent pAKt and Bcl-2 expression levels in both cell types at high SWCNT doses $(\geq 10 \mu \mathrm{g} / \mathrm{ml})$. This suggests that pAkt expression sufficiently regulates $\mathrm{Bcl}$-2-associated anti-apoptotic signaling in B-SWCNT cells [48] at low doses that typically occur in occupational exposures. Under unrealistic high doses, however, B-SWCNT cells may partially succumb to pro-apoptotic intrinsic signaling and may rely on other apoptotic resistance mechanisms (e.g., dysfunctional extrinsic pathway). In summary, prolonged SWCNT exposure coupled with p53 instability resulted in overexpressed pAkt and Bcl-2 which potentially plays a role in the apoptotic resistance and oncogenic development of B-SWCNT cells.

\section{Genes involved in the molecular mechanism of cancer}

Among the various possible molecular mechanisms of cancer, as shown in Additional file 2: Figure S1, both increased (RASGRP, ARHGEF9, SHC, and RHOB) and decreased gene expressions (RBPJK, JNK, DVL1, GSK3A, GSK3 $\beta, P A K, A K T 2$, PMAIP1 [Noxa], BAX) were observed in B-SWCNT cells. Based on overexpressed pAkt, decreased stabilization of p53 via several serine dephosphorylations and increased Bcl-2 protein, it appears that $\mathrm{B}-\mathrm{SWCNT}$ cells gained apoptotic resistance in the intrinsic apoptotic pathway via the pAkt/MDM2/p53/Bcl2 signaling axis. With the decreased pro-apoptotic $B A X$ and PMAIP1 (Table 3, networks 3 and 9) expression downstream of pAkt, and our elevated levels of Bcl-2 protein (Western blot data), we postulated this antiapoptotic signaling mechanism in the intrinsic apoptosis pathway of B-SWCNT cells.

Several DEGs in other signaling pathways potentially contributed to B-SWCNT malignant phenotype. Increased expression of RASGRP1 and RasGEF (ARHGEF9) may contribute to Ras-ERK signaling that regulates cyclin expression and thus cell cycle progression (Table 3, network 1). Increased $S H C$ and Rho $(R H O B)$ expression may also activate $\mathrm{Cdc} 42$, a key cell cycle regulator, and contribute to PI3K/Akt activation. It was reported that smoke-concentrated medium exposure induced Cdc42 translocation in human bronchial epithelial cells that may contribute to lung carcinogenesis [58]. Downregulation of Dsh (DVL1; Table 3, network 3) may remove its blockage of Notch1, which could trigger notch signaling-mediated tumorigenesis [59] and p53-dependent carcinogenesis [60]. Decreased GSK3A and GSK3 $\beta$ expression (Table 3, networks 3 and 9) potentially enhanced $\beta$ catenin tumor promotion and increased Cyclin D protein levels leading to enhanced G1 phase transition and accelerated cell proliferation. It is believed that GSK- $3 \beta$ regulates Wnt signaling pathway and its aberrant activation often results in tumor formation [52]. In this study, downregulated GSK3 $\beta$ in APC complex (a prominent anti-lung cancer mediator), at the transcript or post-transcriptional level via pAkt activation, may lead to dysfunctional $\beta$ catenin and thus potential elevated Wnt signaling (Figure 4). Lastly, downregulation of JNK, a MAPK family kinase, does not seem to support Fos/Jun-mediated transcription in 
A

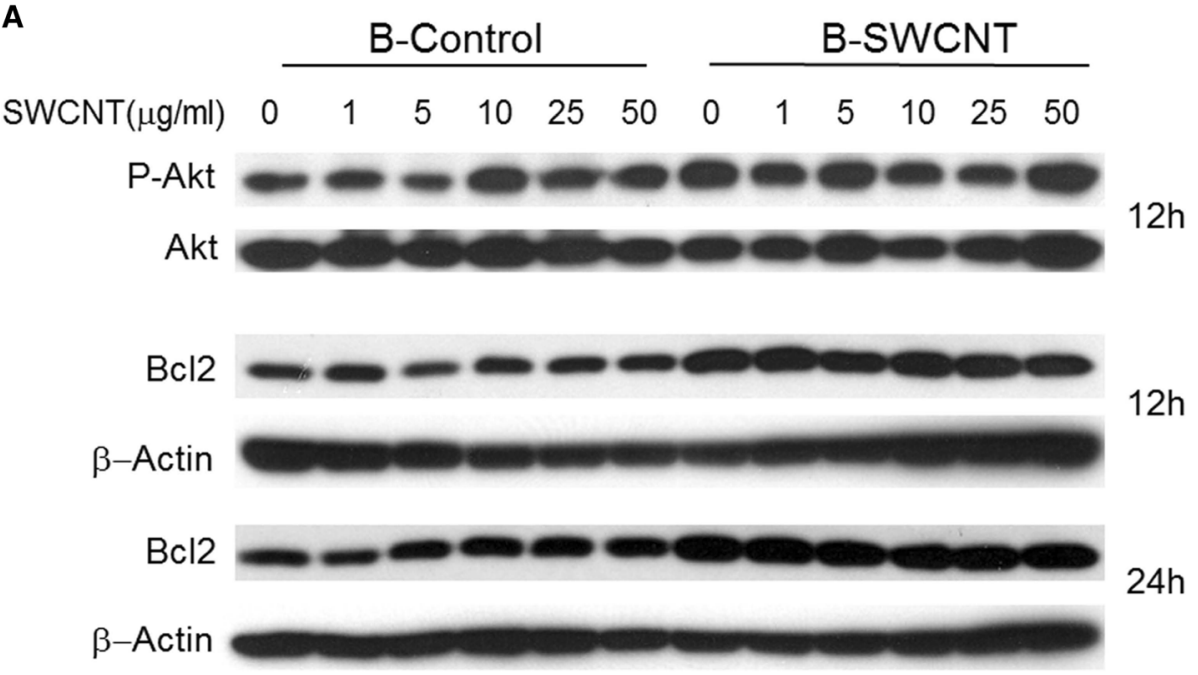

B

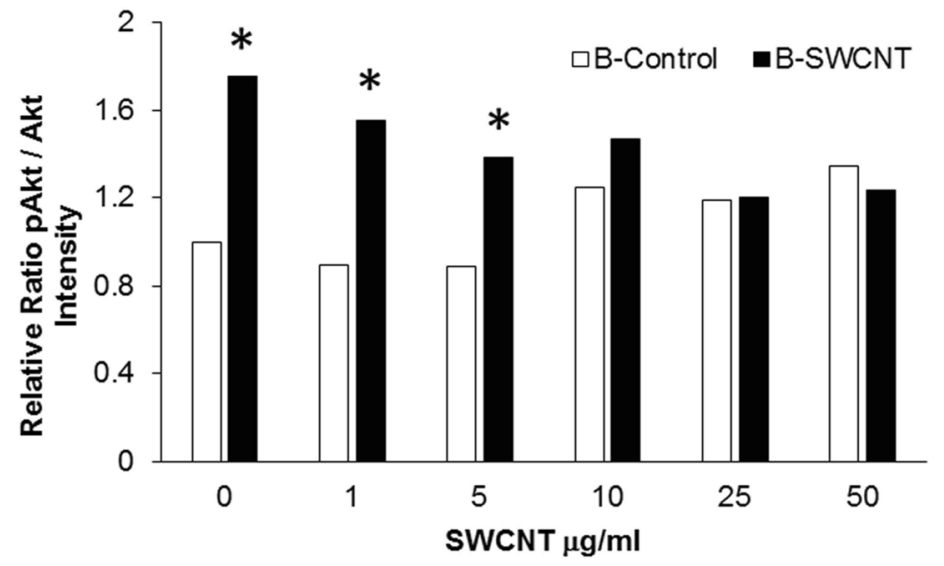

C

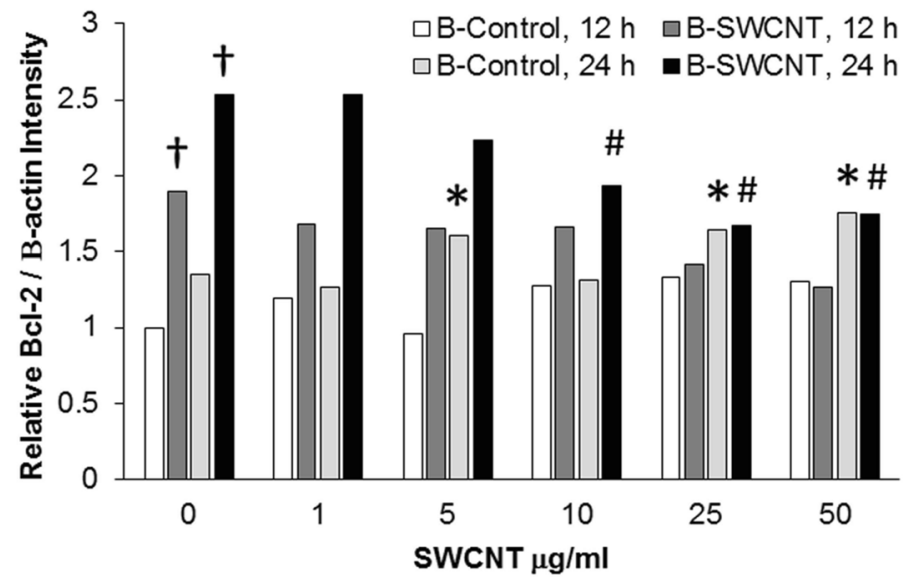

Figure 4 pAkt, Akt, and Bcl-2 expression via SDS PAGE in B-Control and B-SWNCT transformed cells. pAkt, Akt, and Bcl-2 expression via SDS PAGE in B-Control and B-SWNCT transformed cells following 12 and $24 \mathrm{~h}$ re-exposure to dispersed SWCNT (1 to $50 \mu \mathrm{g} / \mathrm{ml}=0.1 \mathrm{mo} 5.2 \mu \mathrm{g} / \mathrm{cm} 2$ ). $\beta$-actin was used as an internal loading control. (A) Representative blots are shown from three independent experiments. (B) pAkt/Akt ratio densitometry quantification. ${ }^{*}$ indicates significant difference between the two cell types $(p<0.05)$. (C) BCl-2 expression densitometry quantification. † indicates significant difference between B-SWCNT and B-Control at each time point $(p<0.05)$. *and \# indicate significant difference of both B-Control and B-SWCNT cells, respectively, compared to its unexposed treatment group at $24 \mathrm{~h}(p<0.05)$. 
SWCNT carcinogenic potential. However, decreased JNK can result in decreased phosphorylation of p53 Ser15, resulting in MDM2-mediated p53 degradation [61].

\section{Conclusions}

In conclusion, we observed that long-term exposure in vitro (6 months) to SWCNT caused malignant transformation of human lung epithelial cells as suggested by our genome-wide expression data. Several genes involved in the apoptosis, cell cycle control, and oncogenic development were altered by the SWCNT treatment with the immune response representing the most altered biological process. The oncogenic phenotype of transformed B-SWCNT cells [13] may be explained, among other possible mechanisms, by active pAkt signaling, destabilized p53, increased expression of Ras family genes, Dsh-mediated Notch1, overexpressed Bcl-2 and downregulation of the anti-apoptotic genes BAX and PMAIP1. The development of the sub-chronic in vitro exposure model coupled with global transcriptome analysis described in this study could aid in the investigation of the potential mechanisms of CNT carcinogenesis, while the genomewide expression approach can provide new insights into the genes involved in the carcinogenic process [40].

\section{Additional files}

Additional file 1: Table S1. Layout of apoptosis protein expression array (R\&D Systems).

Additional file 2: Figure S1. Differentially expressed genes in the molecular mechanism of cancer pathway in SWCNT-transformed BEAS-2B cells. Colors indicate under-(green) and over-expressed (in red) genes compared to unexposed passage control cells. Values are fold changes of SWCNT-treated vs. control, $p \leq 0.05$. The double colors indicate both increased and decreased genes within the same group (e.g., kinases).

\begin{abstract}
Abbreviations
CNTs: carbon nanotubes; SWCNT: single-walled carbon nanotubes; MW: multi-walled; SW: single-walled; B-SWCNT: transformed BEAS-2B cells following SWCNT exposure; B-Control: passage matched, unexposed BEAS-2B cells; DEGs: differentially expressed genes; GO: gene ontology; IPA: Ingenuity Pathway Analysis; ROS: reactive oxygen species.
\end{abstract}

\section{Competing interests}

The authors declare that they have no competing interests.

\section{Authors' contributions}

DC performed data analysis, annotation, and manuscript preparation. TS did the experimental design and manuscript preparation. SL and YL performed the experiment and data collection. YR did the experimental design, interpretation, and manuscript preparation. LW was the study principle investigator for experimental design, data collection, analysis, interpretation and did the manuscript preparation. All authors contributed to preparation of this manuscript, comments and proof reading. All authors read and approved the final manuscript.

\section{Acknowledgements}

This work was partially supported by the National Institute for Occupational Safety and Health, NORA/NTRC and by grants from National Institutes of Health (R01HL095579 and R01-ES022968) and National Science Foundation (EPS-1003907 and CBET-1434503). Authors thank Dr. Robert Oster for helpful discussion and Kai Wu at Wayne State University for the Western blot data analysis assistance.

\section{Disclaimer}

The findings and conclusions in this report are those of the authors and do not necessarily represent the views of the National Institute for Occupational Safety and Health. Mention of brand name does not constitute product endorsement.

\section{Author details}

${ }^{1}$ Division of Preventive Medicine, Department of Medicine, University of Alabama at Birmingham, Birmingham, AL 35294, USA. ${ }^{2}$ Pathology and Physiology Research Branch, National Institute for Occupational Safety and Health, Morgantown, WV 26505, USA. ${ }^{3}$ Department of Pharmaceutical Sciences and Mary Babb Randolph Cancer Center, West Virginia University, Morgantown, WV 26506, USA. ${ }^{4}$ Department of Pharmaceutical Sciences, Wayne State University, Detroit, MI 48201, USA.

Received: 2 October 2014 Accepted: 23 December 2014 Published online: 27 January 2015

\section{References}

1. Zhang Z, Yang X, Zhang Y, Zeng B, Wang S, Zhu T, et al. Delivery of telomerase reverse transcriptase small interfering RNA in complex with positively charged single-walled carbon nanotubes suppresses tumor growth. Clin cancer Res. 2006;12:4933-9.

2. Roco MC. Environmentally responsible development of nanotechnology. Environ Sci Technol. 2005;39:106A-12.

3. Helland A, Wick P, Koehler A, Schmid K, Som C. Reviewing the environmental and human health knowledge base of carbon nanotubes. Environ Health Perspect. 2007;115:1125-31.

4. Jaurand MC, Renier A, Daubriac J. Mesothelioma: Do asbestos and carbon nanotubes pose the same health risk? Part Fibre Toxicol. 2009;6:16.

5. Manning CB, Vallyathan V, Mossman BT. Diseases caused by asbestos: mechanisms of injury and disease development. Int Immunopharmacol. 2002;2:191-200.

6. Song Y, Li X, Wang L, Rojanasakul Y, Castranova V, Li H, et al. Nanomaterials in humans: identification, characteristics, and potential damage. Toxicol Pathol. 2011;39:841-9.

7. Mercer RR, Hubbs AF, Scabilloni JF, Wang L, Battelli LA, Schwegler-Berry D, et al. Distribution and persistence of pleural penetrations by multi-walled carbon nanotubes. Part Fibre Toxicol. 2010;7:28.

8. Mercer RR, Scabilloni J, Wang L, Kisin E, Murray AR, Schwegler-Berry D, et al. Alteration of deposition pattern and pulmonary response as a result of improved dispersion of aspirated single-walled carbon nanotubes in a mouse model. Am J Physiol Lung Cell Mol Physiol. 2008;294:L87-97.

9. Shvedova AA, Kisin E, Murray AR, Johnson VJ, Gorelik O, Arepalli S, et al. Inhalation vs. aspiration of single-walled carbon nanotubes in C57BL/6 mice: inflammation, fibrosis, oxidative stress, and mutagenesis. Am J Physiol Lung Cell Mol Physiol. 2008;295:L552-65.

10. Wang L, Mercer RR, Rojanasakul Y, Qiu A, Lu Y, Scabilloni JF, et al. Direct fibrogenic effects of dispersed single-walled carbon nanotubes on human lung fibroblasts. J Toxic Environ Health A. 2010;73:410-22.

11. Sargent LM, Porter DW, Staska LM, Hubbs AF, Lowry DT, Battelli L, et al. Promotion of lung adenocarcinoma following inhalation exposure to multi-walled carbon nanotubes. Part Fibre Toxicol. 2014;11:3.

12. Sargent LM, Hubbs AF, Young SH, Kashon ML, Dinu CZ, Salisbury JL, et al. Single-walled carbon nanotube-induced mitotic disruption. Mutat Res. 2012;745:28-37.

13. Wang L, Luanpitpong S, Castranova V, Tse W, Lu Y, Pongrakhananon V, et al. Carbon nanotubes induce malignant transformation and tumorigenesis of human lung epithelial cells. Nano Lett. 2011;11:2796-803.

14. Folkmann JK, Risom L, Jacobsen NR, Wallin H, Loft S, Moller P. Oxidatively damaged DNA in rats exposed by oral gavage to C60 fullerenes and single-walled carbon nanotubes. Environ Health Perspect. 2009;117:703-8.

15. Lindberg HK, Falck GC, Suhonen S, Vippola M, Vanhala E, Catalan J, et al. Genotoxicity of nanomaterials: DNA damage and micronuclei induced by carbon nanotubes and graphite nanofibres in human bronchial epithelial cells in vitro. Toxicol Lett. 2009;186:166-73.

16. Yamashita K, Yoshioka Y, Higashisaka K, Morishita Y, Yoshida T, Fujimura M, et al. Carbon nanotubes elicit DNA damage and inflammatory response relative to their size and shape. Inflammation. 2010;33:276-80.

17. Pacurari M, Yin XJ, Zhao J, Ding M, Leonard SS, Schwegler-Berry D, et al. Raw single-wall carbon nanotubes induce oxidative stress and activate 
MAPKs, AP-1, NF-kappaB, and Akt in normal and malignant human mesothelial cells. Environ Health Perspect. 2008;1 16:1211-7.

18. Poland CA, Duffin R, Kinloch I, Maynard A, Wallace WA, Seaton A, et al. Carbon nanotubes introduced into the abdominal cavity of mice show asbestos-like pathogenicity in a pilot study. Nat Nanotechnol. 2008:3:423-8.

19. Takagi A, Hirose A, Nishimura T, Fukumori N, Ogata A, Ohashi N, et al. Induction of mesothelioma in p53+/- mouse by intraperitoneal application of multi-wall carbon nanotube. J Toxicol Sci. 2008;33:105-16.

20. Sargent LM, Shvedova AA, Hubbs AF, Salisbury JL, Benkovic SA, Kashon ML, et al. Induction of aneuploidy by single-walled carbon nanotubes. Environ Mol Mutagen. 2009:50:708-17.

21. Azad N, lyer AK, Wang L, Liu Y, Lu Y, Rojanasakul Y. Reactive oxygen species-mediated p38 MAPK regulates carbon nanotube-induced fibrogenic and angiogenic responses. Nanotoxicology. 2013;7:157-68.

22. Park EJ, Roh J, Kim SN, Kang MS, Lee BS, Kim Y, et al. Biological toxicity and inflammatory response of semi-single-walled carbon nanotubes. PLoS One. 2011;6:e25892.

23. Snyder-Talkington BN, Pacurari M, Dong C, Leonard SS, Schwegler-Berry D, Castranova V, et al. Systematic analysis of multiwalled carbon nanotube-induced cellular signaling and gene expression in human small airway epithelial cells. Toxicol Sci. 2013;133:79-89.

24. Lohcharoenkal W, Wang L, Stueckle TA, Dinu CZ, Castranova V, Liu Y, et al. Chronic exposure to carbon nanotubes induces invasion of human mesothelial cells through matrix metalloproteinase-2. ACS Nano. 2013;7:7711-23.

25. Nagai H, Okazaki Y, Chew SH, Misawa N, Yamashita Y, Akatsuka S, et al. Diameter and rigidity of multiwalled carbon nanotubes are critical factors in mesothelial injury and carcinogenesis. Proc Natl Acad Sci U S A. 2011;108:E1330-8.

26. Shvedova AA, Yanamala N, Kisin ER, Tkach AV, Murray AR, Hubbs A, et al. Long-term effects of carbon containing engineered nanomaterials and asbestos in the lung: one year postexposure comparisons. Am J Physiol Lung Cell Mol Physiol. 2014;306:L170-82.

27. Wang L, Stueckle TA, Mishra A, Derk R, Meighan T, Castranova V, et al. Neoplastic-like transformation effect of single-walled and multi-walled carbon nanotubes compared to asbestos on human lung small airway epithelial cells. Nanotoxicology. 2014;8:485-507.

28. Teeguarden JG, Webb-Robertson BJ, Waters KM, Murray AR, Kisin ER, Varnum $\mathrm{SM}$, et al. Comparative proteomics and pulmonary toxicity of instilled single-walled carbon nanotubes, crocidolite asbestos, and ultrafine carbon black in mice. Toxicol Sci. 2011;120:123-35.

29. Pacurari M, Qian Y, Porter DW, Wolfarth M, Wan Y, Luo D, et al. Multi-walled carbon nanotube-induced gene expression in the mouse lung: association with lung pathology. Toxicol Appl Pharmacol. 2011;255:18-31.

30. Wu P, Yuan SS, Ho CC, Hsieh WY, Hong QS, Yu SL, et al. Focal amplification of HOXD-harboring chromosome region is implicated in multiple-walled carbon nanotubes-induced carcinogenicity. Nano Lett. 2013;13:4632-41.

31. Donaldson K, Murphy FA, Duffin R, Poland CA. Asbestos, carbon nanotubes and the pleural mesothelium: a review of the hypothesis regarding the role of long fibre retention in the parietal pleura, inflammation and mesothelioma. Toxicol Sci. 2010;7:5.

32. She $\mathrm{QB}$, Chen $\mathrm{N}$, Dong Z. ERKs and $\mathrm{p} 38$ kinase phosphorylate $\mathrm{p} 53$ protein at serine 15 in response to UV radiation. J Biol Chem. 2000;275:20444-9.

33. Appella $\mathrm{E}$, Anderson CW. Post-translational modifications and activation of p53 by genotoxic stresses. Eur J Biochem. 2001;268:2764-72.

34. Gerwin BI, Spillare E, Forrester K, Lehman TA, Kispert J, Welsh JA, et al. Mutant p53 can induce tumorigenic conversion of human bronchial epithelial cells and reduce their responsiveness to a negative growth factor, transforming growth factor beta 1. Proc Natl Acad Sci U S A 1992;89:2759-63.

35. Unger T, Sionov RV, Moallem E, Yee CL, Howley PM, Oren M, et al. Mutations in serines 15 and 20 of human p53 impair its apoptotic activity. Oncogene. 1999;18:3205-12.

36. Hussain SP, Amstad P, Raja K, Sawyer M, Hofseth L, Shields PG, et al. Mutability of p53 hotspot codons to benzo(a)pyrene diol epoxide (BPDE) and the frequency of p53 mutations in nontumorous human lung. Cancer Res. 2001;61:6350-5.

37. Stone KC, Mercer RR, Gehr P, Stockstill B, Crapo JD. Allometric relationships of cell numbers and size in the mammalian lung. Am J Respir Cell Mol Biol. 1992;6:235-43.
38. Liao WT, Lin P, Cheng TS, Yu HS, Chang LW. Arsenic promotes centrosome abnormalities and cell colony formation in p53 compromised human lung cells. Toxicol Appl Pharmacol. 2007;225:162-70.

39. Stueckle TA, Lu Y, Davis ME, Wang L, Jiang BH, Holaskova I, et al. Chronic occupational exposure to arsenic induces carcinogenic gene signaling networks and neoplastic transformation in human lung epithelial cells. Toxicol Appl Pharmacol. 2012;261:204-16.

40. Hochberg Y, Benjamini Y. More powerful procedures for multiple significance testing. Stat Med. 1990;9:811-8.

41. Schneider CA, Rasband WS, Eliceiri KW. NIH image to ImageJ: 25 years of image analysis. Nat Methods. 2012;9:671-5.

42. Schindelin J, Arganda-Carreras I, Frise E, Kaynig V, Longair M, Pietzsch T, et al. Fiji: an open-source platform for biological-image analysis. Nat Methods. 2012;9:676-82.

43. Harris CC. p53 tumor suppressor gene: at the crossroads of molecular carcinogenesis, molecular epidemiology, and cancer risk assessment. Environ Health Perspect. 1996;104 Suppl 3:435-9.

44. Lehman TA, Modali R, Boukamp P, Stanek J, Bennett WP, Welsh JA, et al. p53 mutations in human immortalized epithelial cell lines. Carcinogenesis. 1993;14:833-9.

45. Herzog E, Byrne HJ, Casey A, Davoren M, Lenz AG, Maier KL, et al. SWCNT suppress inflammatory mediator responses in human lung epithelium in vitro. Toxicol Appl Pharmacol. 2009;234:378-90.

46. Turabekova M, Rasulev B, Theodore M, Jackman J, Leszczynska D, Leszczynski J. Immunotoxicity of nanoparticles: a computational study suggests that CNTs and C60 fullerenes might be recognized as pathogens by Toll-like receptors. Nanoscale. 2014;6:3488-95.

47. Sargent LM, Reynolds SH, Castranova V. Potential pulmonary effects of engineered carbon nanotubes: in vitro genotoxic effects. Nanotoxicology. 2010;4:396-408.

48. Shivapurkar N, Reddy J, Chaudhary PM, Gazdar AF. Apoptosis and lung cancer: a review. J Cell Biochem. 2003;88:885-98.

49. Gatenby RA, Gillies RJ. A microenvironmental model of carcinogenesis. Nat Rev Cancer. 2008:8:56-61.

50. Park EJ, Zahari NE, Lee EW, Song J, Lee JH, Cho MH, et al. SWCNTs induced autophagic cell death in human bronchial epithelial cells. Toxicol In Vitro. 2014;28:442-50.

51. Zhang T, Qi Y, Liao M, Xu M, Bower KA, Frank JA, et al. Autophagy is a cell self-protective mechanism against arsenic-induced cell transformation. Toxicol Sci. 2012;130:298-308.

52. Behrens J. Control of beta-catenin signaling in tumor development. Ann N Y Acad Sci. 2000:910:21-33. discussion 33-25.

53. Cox ML, Meek DW. Phosphorylation of serine 392 in p53 is a common and integral event during p53 induction by diverse stimuli. Cell Signal. 2010;22:564-71.

54. Ghosh JC, Dohi T, Kang BH, Altieri DC. Hsp60 regulation of tumor cell apoptosis. J Biol Chem. 2008;283:5188-94.

55. Donaldson K, Stone V, Seaton A, Tran L, Aitken R, Poland C. Re: Induction of mesothelioma in p53+/- mouse by intraperitoneal application of multi-wall carbon nanotube. J Toxicol Sci. 2008;33:385. author reply 386-388.

56. Nagai $H$, Toyokuni S. Differences and similarities between carbon nanotubes and asbestos fibers during mesothelial carcinogenesis: shedding light on fiber entry mechanism. Cancer Sci. 2012;103:1378-90.

57. Huang X, Zhang F, Sun X, Choi KY, Niu G, Zhang G, et al. The genotypedependent influence of functionalized multiwalled carbon nanotubes on fetal development. Biomaterials. 2014;35:856-65.

58. Zhang L, Gallup M, Zlock L, Finkbeiner WE, McNamara NA. Rac1 and Cdc42 differentially modulate cigarette smoke-induced airway cell migration through p120-catenin-dependent and -independent pathways. Am J Pathol. 2013;182:1986-95.

59. Pez F, Lopez A, Kim M, Wands JR, Caron de Fromentel C, Merle P. Wnt signaling and hepatocarcinogenesis: molecular targets for the development of innovative anticancer drugs. J Hepatol. 2013;59:1107-17.

60. Licciulli S, Avila JL, Hanlon L, Troutman S, Cesaroni M, Kota S, et al. Notch1 is required for Kras-induced lung adenocarcinoma and controls tumor cell survival via p53. Cancer Res. 2013;73:5974-84.

61. Fuchs SY, Adler V, Pincus MR, Ronai Z. MEKK1/JNK signaling stabilizes and activates p53. Proc Natl Acad Sci U S A. 1998;95:10541-6. 